\title{
Innovative Clinical Assessment Technologies: Challenges and Opportunities in Neuroimaging
}

\author{
Gregory A. Miller \\ University of Illinois at Urbana-Champaign
}

\author{
Thomas Elbert \\ University of Konstanz
}

\author{
Bradley P. Sutton and Wendy Heller \\ University of Illinois at Urbana-Champaign
}

\begin{abstract}
The authors review the reasons for the contrast between the remarkable advances that hemodynamic and electromagnetic imaging of the human brain appear capable of delivering in clinical practice in psychology and their very limited penetration into practice to date. Both the heritages of the relevant technologies and the historical orientation of clinical psychology away from biological phenomena are factors. Discussion of some technical aspects and prospects of these methods and recommendations for facilitating clinical use are provided, with an emphasis on fostering the participation of and contribution by practicing clinical psychologists and professionals in related fields lacking a strong grounding in biological measurement.
\end{abstract}

Keywords: electroencephalography, functional magnetic resonance imaging, magnetoencephalography, neuroimaging, optical imaging

Adaptive or not, a change in the brain's software can-because of neuroplastic alterations of the neural substrate-prompt changes in the brain's hardware (Montague, Hyman, \& Cohen, 2004) in ways that may contribute fundamentally to psychopathology (Hyman, 2005). Neuroimaging methods can assess many such functional and structural changes that occur in mental illness as a prelude to treatment or as a means of tracking its impact. If

Gregory A. Miller, Department of Psychology, Department of Psychiatry, and Beckman Institute Brain Imaging Center, University of Illinois at Urbana-Champaign; Thomas Elbert, Department of Psychology, University of Konstanz, Germany; Bradley P. Sutton, Department of Bioengineering and Beckman Institute Brain Imaging Center, University of Illinois at Urbana-Champaign; Wendy Heller, Department of Psychology and Beckman Institute Brain Imaging Center, University of Illinois at UrbanaChampaign.

This article was undertaken while Gregory A. Miller was a guest professor at the University of Konstanz, Germany. The study was supported by Grant R21 DA14111 from the National Institute of Drug Abuse; Grant R01 MH61358, Grant T32 MH14257, and Grant T32 MH19554 from the National Institute of Mental Health; and funding from the University of Konstanz, the German Research Foundation, and the University of Illinois Beckman Institute and Intercampus Research Initiative in Biotechnology. Stimulating conversations with Patrick Berg, Brigitte Rockstroh, and many other colleagues at the University of Konstanz contributed to the ideas in this article. Thanks are also due to Jeffrey $D$. Lewine, Don M. Tucker, and Andrew G. Webb for consultation on some of the issues discussed in this article, Jennifer L. Stewart and Sarah M. Sass for comments on a draft, and Anna S. Engels for help in preparing Figures 1 and 2.

Correspondence concerning this article should be addressed to Gregory A. Miller, Department of Psychology, University of Illinois at UrbanaChampaign, 603 E. Daniel Street, Champaign, IL 61820-6232. E-mail: gamiller@uiuc.edu psychological phenomena are driving the brain (sometimes mediated by psychological factors tuning gene expression, sometimes more directly) and vice versa, it is not feasible to cast any of these players as "underlying" the others (Miller, 1996). Rather, all of these measurement domains should be considered in clinical practice, as has been advocated for decades (e.g., Davis \& Lang, 2003; Kendler, 2005b; Kozak \& Miller, 1982; Lang, 1968). Given that biological measures loom quite large in current research (and in funding of research) on psychopathology, the near absence of biological measures in psychological work with psychopathology is striking. Biological measures have penetrated some other areas of routine clinical practice that have substantial participation by clinical psychologists, such as behavioral medicine. The article considers what has held back biological measurement more broadly in clinical psychology practice and its prospects for progress.

Definitionally, mental illness in an individual is traditionally conceptualized in terms of subjective experience as manifested in, and diagnosed on the basis of, self-report and overt behavior (Kendler, 2005b). Indeed, mental illness is fundamentally a psychological phenomenon, not a biological phenomenon (Miller, 1996). Nevertheless, mental illness can be viewed as a psychological manifestation of maladaptive brain organization or function that arises from an unfavorable interaction of genome and environment, including the ongoing modulation of gene expression by past and current environments. The rapidly growing scientific and popular literature on the human genome unfortunately has promoted the indefensible assumption that genes will eventually provide an essentially sufficient explanation of psychological dysfunction. Compelling arguments against the appealing simplicity of the notion that there could be "a gene for [diagnosis]" are readily available (e.g., Kendler, 2005a), if not widely noted. As is increasingly acknowledged in the literature on behavioral genetics 
in psychopathology, the action is very likely to be in Gene $x$ Environment interactions, not in gene main effects (e.g., Moffitt, 2005; Moffitt, Caspi, \& Rutter, 2006), whereas more direct genetic effects are likely to be at explanatory levels far removed from manifest psychopathology (Gottesman \& Gould, 2003; Kendler, 2005a). Furthermore, the relevant Gene $\times$ Environment interactions are likely to prove difficult to ferret out because much of the environmental contribution will be what behavioral geneticists have called nonshared, specific to the individual (not shared with, say, an identical twin; McGue, 1999; Turkheimer, 2000). Whether or how often or in what ways we will find that the biology is driving the psychopathology or the psychopathology is driving the biology remains to be established (Kendler, 2005b; Miller, 1996). Widiger and Clark (2000) cited several diagnoses for which biological findings have been proposed as compelling evidence for a diagnosis, but they noted that there is no consensus that inclusion of such criteria is justified or useful. What can be emphasized, without naive reductionism, is that an important feature of biology associated with psychopathology is likely to be abnormal cerebral network architecture with altered neural connectivity (Harrison \& Weinberger, 2005; Meehl, 1962), with symptoms observed in language expression, physiology, and overt behavior (Lang, 1978; Miller \& Kozak, 1993). Conventional diagnosis, such as International Classification of Diseases and Diagnostic and Statistical Manual of Mental Disorders classifications based on rating scales that focus on verbal report and overt behavior, is fundamentally limited by largely overlooking biological data.

It is commonplace to comment on recent progress in brainimaging technologies with great enthusiasm, particularly in claims that we can now do things we could not do recently. Such claims are almost always false, in that most of the progress is much more incremental. It has been possible, for example, to image brain function noninvasively for decades, even in the strict sense of image meaning a three-dimensional representation. What has changed in recent years is that impressive recording technology that was once very rare is now becoming common and better developed, with substantial statistical advances, enabling not only many more but a wider variety of sophisticated (and less sophisticated) users to use it and to help push it forward. This technological growth will continue. It is not enough to ask when, say, functional magnetic resonance imaging (fMRI; the identical method is sometimes called fMRT for tomography) will be ready for routine clinical use. Methods such as diffusion tensor imaging, optical imaging, and transcranial magnetic stimulation are newer still, providing qualitatively different information and possibilities for intervention. We can count on new technologies to continue to appear and some of them to move into routine clinical application.

Even so, technologies take time to mature. The path from idea (or serendipidity) to working implementation to reliable tool to standardized clinical application is neither monotonic nor straightforward. Progress develops in multiple threads in parallel, each thread feeding on others. Across this maturational process from initial research to clinical application, many cooks alter the broth in unforeseeable ways. Sometimes the initial developer of a technology stumbles on a phenomenon outside his or her primary area of expertise while pursuing something more familiar. A result may be atypical expertise creatively brought to bear early in the development process. This fosters novel directions and applications but risks reinventions of the wheel. For example, the seminal publi- cation of the concept of event-related fMRI by Dale and Buckner (1997) surprised many in the electroencephalography (EEG) community because the key elements of the method and its critical importance in experimental design had become the consensus 25 years earlier in the EEG literature (e.g., Hillyard, Squires, Bauer, \& Lindsay, 1971). It was extremely valuable that the authors (one with an EEG/magnetoencephalography [MEG] background) brought this to the attention of the fMRI community, but it is also striking that it needed to be said.

This unfortunately intermittent cross-fertilization between more and less mature methods is reflected in the human FMRI literature more broadly during its short history. Until very recently, the necessary hardware was housed almost exclusively in radiology departments, where much of the clinical emphasis and technological firepower historically have been focused on anatomy rather than function, with virtually no involvement of psychologists. As a result, expertise in experimental design critical for studies of cognitive and emotional function and dysfunction was initially thin, with predictable consequences for the long-term substantive significance of much of the early work. This must be framed, however, not as a failure of the fMRI field but as a normal characteristic in the development of any young field. Clinical and cognitive psychologists as well as basic neuroscientists, steeped in methods for addressing function and dysfunction, are increasingly involved in $\mathrm{fMRI}$ research. The relatively small literatures on brain source localization via EEG and MEG in clinical practice are also growing rapidly, with extensive participation by and impact on clinical, cognitive, and biological psychology. This crossdisciplinary participation bodes very well for, and is indeed a prerequisite to, bringing advanced biological methods into the clinic. Rather than wonder why the physicists and radiologists are taking so long, it is time for clinical psychologists to go after the technology.

\section{Barriers to Neuroimaging in Clinical Psychology Practice}

A thesis of the present article is that professional culture, technological heritage, and misperception of the methodology are major factors in the slowness (Carey, 2005) with which hemodynamic neuroimaging is approaching routine clinical application. A second thesis is that a somewhat distinct set of cultural and technical barriers has held back electromagnetic neuroimaging from widespread clinical use, even though the basic technology has been available much longer. A concerted effort is needed to move these genuinely exciting methodologies into common clinical use, to educate the field on what that use will depend on, and to persuade sufficient numbers of direct service professionals to learn enough about the methods to use them well.

Unlike some of the other methods reviewed in this issue of Psychological Assessment, for the most part neuroimaging based on electromagnetic phenomena (EEG and MEG) and especially hemodynamic phenomena (sensitive to changes in local vascular function, including blood oxygenation and blood flow) is not at a point where it is useful for routine, individual clinical diagnosis or intervention. Besides further innovation and development in clinical research (both substance and method), there needs to be innovation in the perspectives taken by neuroimaging researchers, inspired by input and participation from practicing clinicians guiding the field to ask the questions and develop the approaches that 
will have clinical applications. In part, the goal of this article is to give clinicians some introduction to current issues with these technologies, to put them in a better position to generate ideas about ways in which their needs and perspectives could intersect with neuroimaging and thereby push the field forward, rather than waiting for technologists, often lacking expertise in the phenomena and needs of clinical psychology, to deliver a usable product.

Factors fostering and limiting movement along the application development path are sometimes structural. As noted above, until recently human MRI equipment was almost exclusively the province of radiology departments in larger hospitals, economically justified by and dependent on billable (and thus highly standardized and circumscribed) patient assessments. Research time on such equipment is typically available only sporadically and at times inconvenient for human research subjects (e.g., late at night when hospital demands wane) and ever at risk for interruption by an emergency case bumping researchers off the scanner. In contrast, EEG labs have long been available in most sizable psychology departments, reflecting their enormously lower cost and considerable maturity (brain electrical activity was reported by Caton in 1875, and the first EEG work in humans was published by Berger in 1929). Thus, although EEG is also available in most hospitals, research using it (including research on clinical applications) has not depended on the ability of hospitals to open their doors to researchers. This availability has provided decades of exposure to students and researchers and, not surprisingly, considerable maturity at various levels of the methodology. Nevertheless, the state of the art in EEG is largely unavailable to clinicians. Recent progress in EEG research and applications has emphasized two trends: greatly increased channel count (number of recording locations on the scalp; more channels means better source localization), and more sophisticated source-localization software, the latter demanding considerable technical expertise of users. Other facets of EEG signal processing also have seen considerable progress, although this work is often so dependent on specialized expertise that it is not widely available to (or even seen by) the mainstream clinical research community, let alone filtering into routine clinical application.

The past few years have seen the founding of human MRI sites outside of medical settings, devoted to research and charged with facilitating access by diverse users. This trend will surely accelerate and expand to include dense-array EEG, MEG, optical imaging, and other methods. Eventually, such methods will be nearly as available as traditional low-density EEG. This trend is still a large step away from routine clinical application, but a mature research base is a critical prerequisite.

\section{EEG and MEG: A Mature Technology Transforming and a Young Technology Maturing}

EEG is the most mature of the brain imaging technologies. EEG is typically analyzed as either ongoing oscillatory activity (often measured at rest or with steady-state stimuli) or discrete phaselocking of these oscillations to the processing of specific events, resulting in the event-related brain potential (ERP), sometimes call the evoked potential or evoked response. ERP components are now considered to result primarily from changes in phase-locking of ongoing oscillations reflecting extracellular currents (Makeig et al., 2004). MEG signals originate from intracellular current flow, driven mostly by excitatory postsynaptic potentials (EPSPs) that represent a local intracellular source of current. At different locations, the current penetrates through the neural membrane, such that the circuit can be closed over the volume conductor (i.e., by current pathways through extracellular body tissue). The bioelectric potentials that originate from the volume currents, via EEG or ERP, were integrated into clinical diagnostics decades ago (neurology, audiology, ENT, ophthalmology) and, furthermore, have become fundamental for research in cognitive and behavioral neuroscience, although their use in other contexts of interest to clinical psychologists such as psychopathology remains very limited. Picton (1991) reviewed the already well-established use of auditory ERPs in clinical audiology. Swick (2005) provided a review of the application of ERP assessment with neuropsychological samples, suggesting that it is particularly useful for individuals with aphasia, and resting oscillatory EEG is routinely used in assessment of epilepsy. Dense-array EEG appears to be a promising assessment tool for problems in multisensory integration in autism (Saron, Horton, \& Rivera, 2005)

The widely held assumption that the high temporal resolution of EEG is compromised by poor spatial resolution rests on some misunderstandings of the technology. It is true that, in principle, the measurement of electric and magnetic activity outside a volume conductor, such as the body, does not allow a confident inference about the position and strength of the neural source or sources generating the scalp-recorded activity. In terms of images, the neural activity essentially delivers to the surface a twodimensional shadow of three-dimensional activity. As a consequence, three-dimensional imaging of the sources, the active neural generators, becomes an attempt to find a solution to a set of electromagnetic modeling equations that, by its nature, is not unique. Solving this so-called inverse problem is a challenge, because an infinite number of source configurations within the brain can produce exactly the same distribution of electromagnetic activity on its surface (the "forward problem" is much easier to solve: Given known generators, compute what we will see electromagnetically at the scalp). However, if additional information is provided that constrains the solution space, only one inverse solution may remain that best satisfies the equations. Thus, the spatiotemporal distribution of inferred electromagnetic generators becomes unique, and the inverse problem is solved.

Several types of constraints are available to facilitate the sourcelocalization process. First, there is often a considerable literature on relevant brain areas in a given disorder or a given task. Second, with MRI machines becoming more common in hospitals, and especially with research-dedicated imaging facilities being developed outside of hospital settings, it is increasingly feasible to obtain a structural MRI (sMRI) for each subject. Third, there is progress on the basic research foundations for assumptions about the likely shape and spatial extent of a given neural source and about the electrical conductivity of various brain structures that might distort the signal recorded at the scalp surface. The simplest assumption is that the active brain tissue for a given neural source behaves like a single point of activity, which can be modeled with good approximation by a current dipole (essentially a battery), regardless of the real shape of the neuronal tissue. Straightforward arithmetic predicts scalp activity from such a source, and the fit of that prediction to the obtained data provides a reasonable justification for the model, as it seems unlikely that any other combina- 
tion of sources, by chance, would produce a highly dipolar field pattern.

Whereas any desired temporal resolution in the measurement of magnetic fields and electric potentials of the brain is available by adjustment of the sampling rate, the spatial resolution will generally be limited by the number of channels. Slotnick (2005) reviewed several studies of ERP source-localization accuracy using implanted sources and found striking consistency. Even though none of the studies used what would now be considered a high channel count, every study reported average localization accuracy between 10 and $13 \mathrm{~mm}$. Even more impressive is that these studies used a spherical head model rather than the more accurate, subjectspecific head models that sMRI or surface digitization makes possible. In another review, Gençer, Acar, and Tanzer (2003) cited errors of 10-30 mm but discussed the potential for improvements using realistic head models. With just a 37-sensor array, Mosher, Spencer, Leahy, and Lewis (1993) showed that EEG and MEG resolution could be between 5 and $15 \mathrm{~mm}$ at best. In combination, EEG and MEG reached 2-mm resolution in superficial cortex and were within $10 \mathrm{~mm}$ for a source as deep as $50 \mathrm{~mm}$. In skull phantom work, Leahy, Mosher, Spencer, Huang, and Lewine (1998) demonstrated an average spatial localization error of $3 \mathrm{~mm}$ with 61-site MEG (122 channels, 2 orthogonal channels at each location) and 7-8 $\mathrm{mm}$ with up to 64-channel EEG across 32 dipoles. The 3-mm figure for MEG was largely the 2.26-mm error associated with sensor location measurement, suggesting that MEG otherwise can be remarkably precise.

Thus, although not widely appreciated, at its best (for a single, superficial, tangential, dipolar source), MEG's spatial localization accuracy even with what is now considered a very low sensor count already exceeds that of standard fMRI in many cases. However, the accuracy of source localization is not identical to the accuracy of separating different, simultaneously active sources. For the type of information processing likely to be relevant in many mental illnesses, the ability to identify and track multiple, temporally overlapping brain events is likely to be crucial. Whereas the asymptotic localization accuracy lies somewhere around a few millimeters (particularly the relative localization accuracy), the ability to separate several different sources is about one order lower (Elbert, 1998). Often, however, it is possible to restrict the analysis to a brief moment in time, when there is just one dominant source in a given brain region of particular clinical interest. Alternative to emphasizing dipole sources, constraints such as minimum norm (selecting the solution with the smallest global power of electric potential or magnetic field; e.g., Rockstroh, Junghöfer, Elbert, Guodo, \& Miller, 2006) and other approaches to source localization have been suggested. Indeed, quite a number of methods have developed, especially in recent years, making various assumptions and carrying specific benefits and vulnerabilities (for a review of some available analysis methods and commercial products, see Edgar, Stewart, \& Miller, 2005; Huang et al., 2004).

More sophisticated approaches use anatomical constraints (e.g., Dale \& Halgren, 2001). For most electromagnetic data, for example, only current dipoles with an orientation perpendicular to the surface of gray matter contribute to electromagnetic activity on a macroscopic scale. Increasingly sophisticated software has become available that allows the valid reconstruction of the spatial organization of the cerebral cortex from the sMRI for a given subject.
Limiting the possible source space of the functional localization models to this reconstruction provides constraints that may facilitate determination of the extent, position, and strength of cortical sources for every time point (Dale \& Sereno, 1993; Fuchs, Wag ner, Wischmann, Ottenberg, \& Düssel, 1994; Lütkenhöner, Elbert, Menninghaus, Steinsträter, \& Wienbruch, 1996).

The growing impact of appropriate anatomical constraints in electromagnetic neuroimaging cannot be overstated. Three factors are converging to push this forward. First, only recently have EEG channel counts above a dozen or so become reasonably common; therefore, for most of the history of EEG, sophisticated source localization was rarely an option. Second, with sMRI a more recent technology than EEG, and with sMRI largely confined to major medical centers until very recently, it was infeasible for most EEG studies to obtain subject-specific SMRI. With the growing movement to set up research-dedicated MRI machines outside of medical centers in ways that foster access by diverse researchers and with growing channel counts in EEG labs, source localization is becoming far more feasible. The third development is in sourcelocalization software, a relatively young field now rapidly expanding. Conventional wisdom about the poor spatial localization potential of EEG/MEG relative to fMRI rests in part on an unappreciated discrepancy that is rapidly becoming obsolete: fMRI studies always (and quite easily) collect sMRI data, whereas EEG/MEG studies rarely do (though this is now changing). Thus, most of the literature bearing on the relative spatial accuracy of EEG and fMRI actually pits EEG/MEG (without sMRI) against fMRI (with sMRI), clearly not an informative comparison. As sMRI becomes increasingly available to EEG/MEG researchers, this discrepancy will fade. Indeed, rather than comparison between methods, integration of them is clearly where the field needs to go (e.g., Brass, Ullsperger, Knoesche, von Cramon, \& Phillips, 2005; Dale \& Halgren, 2001; Miller et al., 2004). Efforts are now underway to develop EEG recording methods usable in a magnetic resonance (MR) scanner, which will further accelerate this integration.

There is no question that the spatial localization performance of EEG and MEG, an active area of development, will continue to improve. Optimistic predictions notwithstanding, Srinivasan (2005) noted that localization accuracy depends greatly on the nature of the signals being localized. A mixture of local, regional, and global sources probably contributes to the measured EEG and MEG (Nunez, 2000; Srinivasan, 2005). Scherg, Ille, Bornfleth, and Berg (2002) argued that spatial localization is often a less valuable contribution of EEG or MEG analysis than identifying the number and time courses of active sources. For example, using 61-site (122-channel) MEG, Huang et al. (2003) showed that $97 \%$ of the variance in nonpatients' P50 auditory ERP component could be accounted for by a bilateral pair of sources localized to the superior temporal gyrus. The same analysis accounted for a still substantial but very significantly smaller $86 \%$ of the variance in a schizophrenia comparison group. Furthermore, the residual variance in the patient group was not, as one might anticipate, largely noise. Rather, it showed a very clear $40-\mathrm{Hz}$ oscillation, suggestive of a third source characteristic of the patient group. These data exemplify not only the potential real-world effectiveness of MEG source localization for well-defined sources in well-developed paradigms but the generative value of time-course information even when not all possible sources have been localized. 
Although at present relatively few clinical or research EEG labs have as many as 64 channels available, 64- and 128-channel systems are becoming more common, particularly in newly established labs. This capability is in stark contrast to the longstanding norm of a 19-channel montage still used for routine clinical EEG assessments in medical contexts and still lower channel counts long typical in cognitive research. Luu et al. (2001) provided a practical, accessible review of the importance of high channel counts for localization in neurological and neurosurgical practice. In recent years, the state of the art has advanced to provide 256 EEG channels (512-channel development is now being evaluated by at least two companies), and 275 and 306 MEG channels are now available in commercial MEG systems (although advertised channel counts are sometimes misleading, as some systems colocate sensors of different types or orientations). Most EEG and MEG work is still conducted successfully with far lower channel counts, but the densest arrays are certainly of value in many contexts, and dense arrays are clearly where the field is headed. Srinivasan (2005) suggested $2 \mathrm{~cm}$ as the ideal distance between EEG sensors.

The spatial density of EEG and MEG sensor placement has gotten considerable press, but often a far more important consideration is the extent of coverage over the entire head. As the channel count has increased, coverage has typically extended to lower and lower portions of the head. For biophysical reasons, this is critically important (Junghöfer, Elbert, Tucker, \& Braun, 1999). As noted above, for many research questions and clinical applications, precise anatomical location of the neural sources is far less important than differentiation of sources and identification of their time courses. This is particularly true for the growing focus on functional connectivity in brain circuits, where the gross anatomy is often known, and identifying the general regions that are active during a task is not too difficult with behavioral and hemodynamic methods, but the relative time courses of activity in various parts of the circuit-critical to sorting out the causal relationships-is poorly addressed by such methods. Invasive and noninvasive EEG is making great strides in this area. MEG is equally and potentially even more capable, though it has faced other obstacles.

MEG has followed a trajectory different from the other methods considered here. Available (Cohen, 1968; as a demonstrated method of spatially localizing imaging: Brenner, Lipton, Kaufman, \& Williamson, 1978) about as long as SMRI (Lauterbur, 1973) and considerably longer than fMRI (Ogawa, Lee, Kay, \& Tank, 1990), MEG shares the high cost (in hardware and in signal processing) of MRI but not, to date, its broadly established clinical billability (primarily for sMRI, not fMRI). The clinical utility of MEG has nevertheless been established for some time (e.g., Lewine, Andrews, et al., 1999; Lewine, Orrison, Sloan, Kodituwakku, \& Davis, 1999; Mäkelä et al., 2001; Orrison \& Lewine, 1993). For example, MEG has been a superb tool for years in some presurgical planning cases for epilepsy (Moore, Funke, Constantino, Katzman, \& Lewine, 2002; J. R. Smith et al., 1995; Vieth, Kober, Ganslandt, Möller, \& Kamada, 2003), including pediatric cases (Verrotti et al., 2003; Wolff et al., 2005), and evidence is emerging of clinical utility even in mild head trauma (e.g., Lewine et al., in press). MEG systems are comparatively rare in the United States (relative to Japan and western Europe), although increasing approval by insurers of a few clinical assessment protocols has led to a recent acceleration of United States installations, with some systems' business model relying solely on clinical income. The U.S. federal standard reimbursement for clinical MEG has varied considerably by year but for 2006 is US $\$ 2750$ for epilepsy localization, with typical billings much higher because of multiple procedures per session (VSM MedTech, 2005).

Arguably, the primary factor holding back clinical application of MEG is limited access: There are relatively few systems. Secondarily, commensurate with the low number of MEG installations, the expert user community is comparatively small. Although a small community of well-qualified leaders is moving the technology forward, there is not the large, established base of EEG users or the flourishing growth of fMRI users who, although not all as expert, play a critical role in translational research, moving the technology into the clinic. Other than an improving case for the economics of medical use of MEG, the most promising trend for MEG's prospects is the growing realization that EEG and MEG will provide vital complements to hemodynamic imaging, largely subsequent to the case for integration made by Dale and Halgren (2001). As a result, analysis methods are beginning to converge, such as enhancements to the popular Statistical Parametric Mapping package, developed for hemodynamic imaging, that now handle EEG and MEG data as well (Kiebel, Tallon-Baudry, \& Friston, 2005).

\section{fMRI: A Young Technology Maturing Rapidly}

With their appealing images, first positive emission tomography (PET) and now FMRI have unquestionably captured the public imagination and received the lion's share of neuroimaging headspace in the past decade. That fMRI is strikingly primitive is commonly overlooked in the popular press, yet should be understood to mean that it has a great future ahead as it grows out of its infancy. For example, to date, fMRI has been hobbled by vulnerability to large spatial distortions associated with inhomogeneities in magnetic susceptibility among adjacent tissues (a serious problem for some but not all brain regions of special interest to psychologists, such as the amygdala and orbitofrontal cortex; Figure 1). However, overcoming this problem has attracted a number of research programs. MR scanners typically distort areas of frontal and medial temporal lobes and other regions near airtissue interfaces in and around the brain. A standard analysis step known as registration (moving or stretching the image to align with some template) can extend this distortion to other regions as well, but field inhomogeneity correction methods are under development to address this. These distortions and the state of the art in addressing them are not widely understood and warrant some discussion in order to judge fMRI's current status and potential.

Magnetic resonance imaging developed initially as a way to examine structure, without the ability to look at regionally specific activity (function). Whereas some nuclear magnetic resonance phenomena were discovered decades earlier, the first demonstration of the use of nuclear magnetic resonance to create images of an object (Lauterbur, 1973) eventually led to every major hospital having an MRI machine for clinical assessment of soft tissue structure, such as some kinds of tumors and the arborization of blood vessels. sMRI (often what is meant by the term MRI, although that is ambiguous since the arrival of $\mathrm{fMRI}$ ) is a valuable complement to X-ray methods, which are better for imaging bone structure. Beginning in the early 1990 s, several labs published 

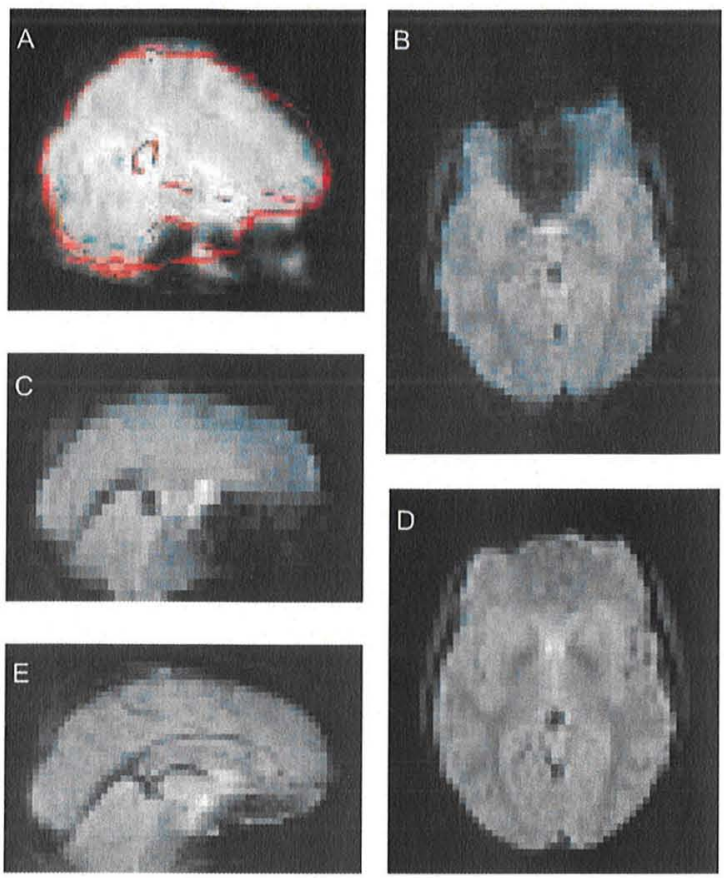

Figure 1. Illustration of actual blurriness of typical fMRI images as well as registration distortion and sometimes severe signal loss due to susceptibility artifact. Data collected at 3T. Panel A shows how an automated registration algorithm can pull the frontral cortex ventrally (away from correct red outline) as a result of lack of signal in the orbitofrontal area, distorting localization not only of the ventral areas but of the entire frontal cortex. Panels B and C show signal loss in the ventral frontal areas due to susceptibility artifact. Panels D and E show the same slices from the same subject rerun with different fMRI acquisition parameters designed to minimize such signal loss and with field-map correction. Adapted from Miller (2005).

demonstrations of adaptations of sMRI in which the signal is dependent on local blood oxygenation, which changes with local neural activity. In blood-oxygenation-level-dependent (BOLD) fMRI, the current standard method, a series of measurements are done that are sensitive to magnetic susceptibility differences between deoxygenated hemoglobin and surrounding tissue. Deoxyhemoglobin can be slightly magnetized relative to surrounding tissue, whereas oxyhemoglobin cannot, and this difference is the basis of the fMRI measurement. However, sensitizing the image acquisition to this difference, by placing the tissue in a combination of fixed and varying magnetic fields (see next paragraph), also sensitizes it to macroscopic magnetic susceptibility differences between tissue, fluid, and air interfaces in and around the brain. These susceptibility differences lead to perturbations in the magnetic field experienced by tissues near interfaces in the human head and can be readily portrayed in simulations (Truong, Clymer, Chakeres, \& Schmalbrock, 2002). The magnetic field inhomogeneity induced by the susceptibility differences can lead to two types of artifacts in fMRI acquisition: geometric image distortion and signal loss. These susceptibility artifacts are major barriers to both research and clinical use of fMRI at present. Both of these types of artifact are active areas of methodological research in
fMRI, and it must be emphasized again that this is a young technology that will surely improve.

The first type of artifact, geometric distortions in the image, depends on the acquisition sequence and specifically on the spatial frequency (or k-space) sampling trajectory. Acquisition refers to the spatial pattern and temporal course of the radio-frequency (RF) pulses used to probe the MR signal in each brain region. In the most common acquisition method used in fMRI, echo planar imaging (EPI), the spatial frequency space is sampled in a rasterlike (line-by-line) pattern. The geometric distortions resulting from field inhomogeneity in EPI are spatial shifts of pixel intensities along the slow or phase-encode direction (perpendicular to each raster line; Sekihara, Kuroda, \& Kohno, 1984). When using spiral sampling as an alternative method of scanning the spatial frequency space, circular blurring of pixel intensities results (Yudilevich \& Stark, 1987). Various methods have been developed to correct for these susceptibility-induced geometric distortions. Most rely on measurement of the magnetic field distribution using two acquisitions with slightly different RF-pulse echo times to form a magnetic field map (Schneider \& Glover, 1991). For EPI acquisitions, this field inhomogeneity can be converted to a pixel offset map and can be used to "translate" (shift spatially) the pixel intensities, moving them closer to their true location in the body (Jezzard \& Balaban, 1995; Reber, Wong, Buxton, \& Frank, 1998; Sekihara et al., 1984; Sumanaweera, Glover, Binford, \& Adler, 1993). When spiral acquisitions or other non-Cartesian sampling trajectories are used, the predominant methods to correct the distortion are conjugate phase (Schomberg, 1999), simulated phase evolution rewinding (Kadah \& $\mathrm{Hu}, 1997$ ), and iterative methods (Harshbarger \& Twieg, 1999; Sutton, Noll, \& Fessler, 2003). The point here is that geometric distortions in the image, although a serious problem, are well understood in the technical literature on fMRI (if not in the broader user community), methods already have been developed to address the problem, and additional work is underway.

The second artifact from magnetic field inhomogeneity is referred to as susceptibility-induced signal voids. Gradients in the magnetic field within a volume element (voxel) cause protons within the voxel, subjected to the scanner's magnetic fields, to dephase and their signals to cancel, resulting in a signal void in the image at the location of the voxel. Because of the geometry of air-tissue interfaces in the brain, especially near the orbitofrontal cortex, most of these methods usually address only z-directed (inferior/superior direction) gradients of the field. This distortion is particularly problematic for clinical applications because the orbitofrontal cortex is believed to be critical in a variety of clinical phenomena, including emotional (especially appetitive) processing relevant to depression, substance abuse, and social judgment. Finding ways to minimize this distortion is an active area of research, and several methods have been proposed to address the signal cancellation. One family of methods uses an imbalanced slice-select gradient as a compensation for the induced magnetic field gradient (Glover, 1999; Yang, Williams, Demeure, Mosher, \& Smith, 1998). Compensation gradients also can be applied as phase gradients in specially tailored excitation RF pulses (Cho \& Ro, 1992). These pulses can have three-dimensional structure to accurately compensate a two-dimensional slice (Stenger, Boada, \& Noll, 2003). In addition to these acquisition-time methods, reconstruction techniques are being developed to examine what infor- 
mation can be obtained if estimates of the gradients are known (Sutton, Noll, \& Fessler, 2004). Finally, methods have been developed to reduce the magnetic field gradient by introducing passive diamagnetic shims that can be placed in the mouth (Wilson \& Jezzard, 2003) or active shim coils that can be dynamically tuned to provide optimal susceptibility gradient cancellation (Hsu \& Glover, 2005).

fMRI signal quality is also subject to physiologically induced susceptibility fluctuations, to subject motion artifact, and to motion-by-susceptibility interactions. An additional major barrier for some kinds of clinical use, though not a signal-quality issue, is temporal resolution. The fMRI signal is typically sampled approximately every $2 \mathrm{~s}$. For many phenomena of clinical interest, this is more than adequate, but for many others it is not. Some trade-offs (such as measuring only a small part of the brain) allow this sample rate to be increased significantly (e.g., one slice every 100 $\mathrm{ms}$ as subjects viewed emotional stimuli; Sabatinelli, Bradley, \& Lang, 2005).

Another technical issue about fMRI that needs to be understood to judge its current status and near-term prospects is that its typical spatial resolution is generally considerably lower than illustrations commonly convey. In most cases, the spatial resolution of the accompanying sMRI is considerably higher than the fMRI on which a given study focuses. It is common to overlay a colorcoded, thresholded fMRI activation map on a coregistered grayscale sMRI map. This coregistration is helpful in conveying the locations of the fMRI activations. However, the visual sharpness accomplished by a combination of thresholded fMRI and highresolution sMRI image implies a degree of spatial precision not available in the fMRI data and made worse by the standard practice of spatial smoothing over approximately 5 to $10 \mathrm{~mm}$ prior to analysis. The rather blurry images of Figure 1 are unthresholded fMRI, with high-field human acquisition parameters providing better spatial resolution than much of the published literature. On the other hand, nonstandard fMRI methods using appropriate surface array coils and nonstandard data processing can achieve local resolution better than $1 \mathrm{~mm}$. Thus, the localization potential for fMRI (distinct from typical current practice of a single coil for the whole head) is quite impressive.

A critical issue for bringing fMRI into the clinic is reproducibility. As with many other kinds of data (including typical EEG and MEG methods), reproducibility at a group level can be addressed at least in principle by using an appropriately large number of subjects for sampling the population. However, most clinical judgments are made about individual patients, and the degree of confidence in the occurrence, amount, or location of a phenomenon must often be quite high to justify an intervention decision. To date, clinically adequate reproducibility has been a significant concern for fMRI. This issue has two facets. First, reproducibility for a single subject is essential for ensuring the validity of a clinical finding (e.g., presurgical localization of function, prior to nearby tissue removal). Second, reproducibility across subjects and across laboratories (and thus across brand and field strength of scanner, acquisition pulse sequences, data analysis methods, etc.) is necessary for the accrual and cross-validation of knowledge in clinical science. The rate of progress in determining and improving reproducibility in fMRI is likely to vary by subject demographics, brain region, activation method, and clinical issue, but several groups are working actively to determine and enhance it (e.g.,
Biomedical Information Research, n.d.; S. M. Smith et al., 2005) and to develop standardized methods of routine image-quality assessment (e.g., Stöcker et al., 2005).

One other issue affecting the readiness of $\mathrm{fMRI}$ for prime time in the clinic is its very richness as an approach to measuring brain activity. There is a huge variety of stimulus and task protocols for which it is appropriate, recently expanding into social psychology (social neuroscience), economics (neuroeconomics), and no doubt unforeseen areas. By contrast with the relatively narrow-range and well-established protocols used in X-ray of a broken forearm or low-density EEG assessment in a standard sleep workup, the fMRI research literature and the human, technical, and financial resources available to develop the technology are very scattered across diverse paradigms. In the long run, thoroughly sampling the protocol space is good for the science, but in the short run, it limits the pace of accrual of the research base around any specific clinical question.

The limitations discussed above are widely understood among those technically proficient in MRI but not among prospective clinical users, journalists, or the general public. This discrepancy fosters premature enthusiasm and then disappointment about what can be delivered now or soon (Carey, 2005). To appreciate how recently fMRI has come to be reasonably available, an assessment just over a decade ago was that only four or five MR systems in the world could provide good-quality fMRI of the human brain (Turner \& Jezzard, 1994). fMRI's trajectory in its short life is remarkable, but it is not the magic bullet often portrayed in the press (or in some grant applications). As noted elsewhere in this article, fMRI will likely prove most valuable as a complement to other imaging methods as well as to neuroscientifically informed behavioral assessment. Public perceptions notwithstanding, its true potential remains to be realized. The slope of real progress is encouragingly steep.

\section{Comparing and Integrating Electromagnetic and Hemodynamic Imaging}

The following pros and cons of electromagnetic source imaging versus hemodynamic measures need to be considered (expanded from Elbert, 1998) to understand their distinct roles and potential integration:

1. Procedures that image blood flow parameters via MR generally require difference maps comparing two conditions. If activity measured during a resting condition is subtracted from that during an active task condition, the resulting blood flow pattern may show where task processing is implemented. It may, however, be determined as much by the type of resting condition (such as lying in a small, noisy tube) or by some nonlinear interaction between the two conditions. Psychologists are well aware that so-called resting or baseline conditions may involve very considerable processing that may vary by individual, diagnosis, and so forth. Thus, selection of conditions to subtract is a critical issue in hemodynamic imaging that often does not get adequate attention. In contrast, in EEG and MEG, it usually suffices to use a local baseline (such as $100 \mathrm{~ms}$ immediately before a stimulus) against which the impact of 
the stimulus is much less ambiguous. Resting oscillatory activity may be revealing without the need for such a subtraction procedure. For example, often in brain damage, abnormal electric and magnetic slow-wave activity is focally generated in the vicinity of the lesion. These generators can be identified by means of MEG, using the simple equivalent dipole model, in patients suffering from stroke-related functional impairment (aphasia and hemiplegia) or psychopathology. Evidence suggests that the distribution of the focal concentration of generators of slow waves distinguishes patients with psychiatric disorders such as schizophrenia, affective disorders, and posttraumatic stress disorders. Research (reviewed by Rockstroh et al., 2001, Rockstroh, Ray, Wienbruch, \& Elbert, 2006, briefly summarized below) suggests that focally generated slow-wave activity indicates dysfunctional neuronal network architecture and communication that may mediate subsequent neuropsychological and psychopathological signs. One implication of this is that focal slow-wave mapping offers a deep-structure assessment of psychopathological processes, including changes following treatment, and may thus provide essential additional information to supplement standard surface-level diagnostic procedures based solely on symptoms observed in overt behavior and verbal report.

2 As is often common with new technologies, the signal one is dealing with in fMRI is relatively weak, with the signal-to-noise ratio much smaller than that available from direct neural recordings (Raichle, 2001). This is generally true throughout the head, whereas for EEG and MEG the recorded signal is somewhat depth-dependent.

3. EEG and MEG are not vulnerable to the magnetic susceptibility problem that for some brain regions is severe for fMRI, as discussed above. Conversely, other than magnetic susceptibility, fMRI's spatial resolution generally does not decline for deeper structures as happens for EEG and MEG.

4. Not every neural mass activity will create the kind of open fields necessary for detection by EEG or MEG, but proper selection of task and analysis procedures may permit electromagnetic source localization at depths and in structures commonly thought to be unreachable. For example, evidence of localization to the hippocampus has been obtained in nonpatient, psychiatric, and neurological samples (Hanlon et al., 2003, 2005; Tesche, 1997), despite using a type of MEG sensor thought to be least sensitive to deep sources and despite the somewhat closed structure of the hippocampus, making it particularly difficult, in principle, to register with remote electromagnetic sensors.

5. The link between blood flow and neural activity is not well understood. Raichle (2001) noted that, "After a century of research, we still do not know how or why blood flow increases during neuronal activation" ( $p$.
130). In response to a pioneering article showing one possible relationship between an fMRI signal and neuronal local field potentials (Logothetis, Pauls, Augath, Trinath, \& Oeltermann, 2001), a simplistic assumption that the relationship is generally linear has become prevalent. Devor et al. (2005) recently cast doubt on the ability of fMRI to resolve neuronal location, showing that the fMRI signal reflects hemodynamic activity extending beyond the relevant active neurons and that the neuronal-hemodynamic relationship can be very nonlinear. Such effects may vary with subject demographics (including age, in part due to vascular changes), brain region, and neuropathology associated with a given type or degree of psychopathology.

6. The relationship between cytoarchitecture and brain morphology is variable. Blood flow integrates neuronal activity over relatively long periods of time and, thus, both prevents monitoring of faster aspects of cognitive processing in real time and precludes study of much of the functional connectivity among brain regions, often realized through brief and rapid changes in the coherence of spatially distributed oscillations of cell assemblies. The sequence of relevant events may unfold at time lags much too brief for hemodynamic measures to distinguish. There are circumstances under which conventional fMRI may achieve better temporal resolution than its long intersample interval would normally permit (e.g., Huettel \& McCarthy, 2001a, 2001b). However, even when $\mathrm{fMRI}$ measurement is limited to a few slices, permitting much more frequent sampling, the temporal blurring and delay inherent in hemodynamic phenomena limit the measures to relatively sustained neural events.

7. The temporal resolution of hemodynamic measures is limited by the fact that the typical hemodynamic response emphasized in fMRI unfolds slowly relative to many brain events, requiring approximately $2 \mathrm{~s}$ to onset, 4 to $6 \mathrm{~s}$ to reach peak amplitude, and approximately $15 \mathrm{~s}$ or more to resolve. The dependence of this time course on brain region, task, and various subject characteristics has not been thoroughly explored. It must be emphasized again, however, that as a young technology fMRI is very likely to improve substantially. For example, Huettel, Song, and McCarthy (2004) discussed a possible means of detecting neuronal activity directly with MRI, though it has yet to be demonstrated in humans. Such an advance could bring MRI into line with the temporal resolution of EEG and MEG. Recent fMRI technology, though not yet widespread, already provides a means of speeding up acquisition (e.g., Knake et al., 2005) and, as noted above, trade-offs that can improve its temporal resolution.

8. Surprisingly, the human fMRI literature in clinical and cognitive neuroscience has rarely included brain region as an explicit factor in analyses. Individual voxels or regions of interest are analyzed separately, often with 
careful attention to experiment-wise error rate, but inappropriate inferences are then drawn about apparent differences (or lack thereof) in effects as a function of location that have not been tested directly. (See Herrington, Koven, Miller, and Heller, 2006, for an extended critique of this problem and its implications for research on emotion and psychopathology.) This limitation in experimental design is paralleled in limitations in popular analysis packages that have not facilitated inclusion of region as a factor.

9. The EEG/MEG literature is similarly hobbled by uncertainty about how to analyze dense-array data. Like interpretation of distributed hemodynamic imaging findings, electromagnetic source localization remains an art as much as a science, particularly challenging for activity manifesting "higher order" cognitive events in which numerous sources may be active more or less simultaneously. Many dense-array studies simply collapse nearby channels together to simplify the analysis, at the cost of regional detail that may be crucial to capturing distinctive brain events.

10. When EEG and MEG are compared to measures of regional cerebral blood flow, it is often mentioned that fMRI and radiologically instrusive methods such as PET and single photon emission computed tomography, but not EEG or MEG, rely on physical principles that allow the unequivocal reconstruction of spatial pattern. In fact, all of these methods require considerable data transformation and assumptions about links of the raw measure to neurophysiological processes, so such a distinction is unconvincing. Depending on a variety of choices made during data analysis, each of these imaging techniques can produce a variety of outcomes from the same original data set. The interpretations also vary with assumptions of what physiological process might have caused the alterations extracted by the signal analysis. It is, therefore, particularly valuable to crossvalidate findings and conclusions, using different imaging methods, sensitive to the strengths and weaknesses of each (i.e., multimodal imaging).

The first study comparing electromagnetic and hemodynamic brain activity (during a verb generation task; Eulitz et al., 1994) demonstrated that MEG results could not be explained by limiting sources to structures that were active in PET and indicated rather that there were sources in primary and secondary auditory cortices, as well as in parietal regions, that were not obvious in the hemodynamic data. This discrepancy could reflect, for example, the relative insensitivity of hemodynamic methods to transient phenomena that have little impact on local metabolism but are nevertheless evident in electromagnetic recordings. It is now widely understood that EEG and MEG sometimes will be able to "see" transient activity invisible to hemodynamic measures because of the rapid time course (and thus low metabolic impact) of some neural phenomena and the at best indirect coupling of hemodynamic events to neuroelectric events.
Although it is fashionable to offer summary statements about the relative temporal and spatial resolution of various recording methods-and we have reviewed some issues and evidence bearing on such comparisons here-it is far less common to discuss what phenomena, at what temporal or spatial resolution, are worth recording. Indeed, comparative statements about resolution often beg the question of what is being measured. Because there are brief electrochemical events with essentially no net metabolic footprint, no improvement in hemodynamic neuroimaging technology is going to be able to match the temporal or spatial resolution of electromagnetic recordings of such events. Conversely, there are neurochemical events that magnetic resonance methods such as MR spectroscopy can detect that leave no net electromagnetic trace visible to distant sensors. For neither of these examples is it meaningful to talk of comparing resolution among these classes of measures, any more than one would characterize how well a light meter measures sound pressure. The increasing diversity of neuroimaging methods provides tools, each better for some purposes than others.

\section{Optical Imaging: A Newcomer With Great Promise}

Least known among promising brain-imaging methods is a class of methods known as optical imaging. The use of near-infrared light is becoming increasingly important for noninvasive biomedical imaging because of its low cost, flexible applicability, and low impact on biological tissue. The basic approach is literally to shine a light through the skull (which is much more transparent to some frequencies than one might imagine) from one or more sources and to record from one or more sensors. By pulsing the light from different sources at different times or by using different light frequencies, each sensor can distinguish the source of a given signal. Photons diffuse through tissue as a function of several factors, with a portion returning to the surface, and intensity and timing can be informative about the neural tissue and neural function in between. Such diffuse photon imaging is presently used in several different ways: (a) Pulsed stimuli with gated detection allow for the discrimination of ballistic photons and a reconstruction of optical density, (b) low-coherence tomography operates via reflection and allows enhanced longitudinal spatial resolution, and (c) diffuse photon density wave imaging uses an amplitudemodulated light source (at $\mathrm{MHz}$ frequencies) and measures amplitude and phase some distance from the source.

The various optical methods require some means of calculating distributions of optical densities within the volume from the measured data (Gratton, Fabiani, Elbert, \& Rockstroh, 2003). Even in the absence of static scattering, contrast information on buried dynamical heterogeneities can be gained from spatially resolved measurements. Optical imaging may even be used noninvasively to image biochemical activity related to information processing in the brain on a subcellular level (Li et al., 2005). In addition to such foreseeable developments, optical imaging appears to be uniquely positioned to connect other imaging methods, particularly electromagnetic and hemodynamic methods (Gratton et al., 2003). Specifically, a particularly appealing feature of optical imaging is that different forms of it are partially redundant with MR measurement (directly sensitive to hemodynamic activity) or with EEG and MEG measurement (directly sensitive to neural activity). As a result, there is considerable interest in optical methods as a way to 
understand neurovascular coupling which, in turn, would likely lead to better understanding of hemodynamic and electromagnetic data. As with EEG and MEG, the use of higher and higher density recording arrays is growing in optical imaging. The July 2003 issue of Psychophysiology (Bradley, 2003) can be consulted for a series of articles on optical imaging and its prospects.

\section{Clinical Translation of Neuroimaging Research}

It is clear, and belatedly beginning to be appreciated, that much of the future of neuroimaging lies in multimethod integration (Dale \& Halgren, 2001). For example, fMRI as currently deployed directly measures hemodynamic rather than neural activity, whereas EEG and MEG measure neural activity directly. The optical signal shows promise as a means to record both hemodynamic and neural activity simultaneously and directly, though it is probably confined to superficial regions. MEG is less sensitive than EEG to radial sources (Mosher et al., 1993), making MEG particularly good for registering sulcal activity, whereas EEG will tend to do better with gyral activity. MEG is less vulnerable to inhomogeneities in tissue impedance than EEG, and EEG and MEG do not suffer from the magnetic susceptibility artifact that is problematic in fMRI. EEG, even in dense-array configurations, is much less expensive than MEG or MRI. sMRI will prove to be as invaluable in registering EEG and MEG signals to subject-specific brain structure as it does for fMRI, and fMRI will provide an important cross-check to functional localizations, as will optical imaging for superficial activity.

In clinical application, unfortunately, this multimodal approach will face the same obstacles discussed above: the cost of equipment, the cost of advanced technical expertise in collecting and interpreting such data, the steep challenge of any one individual mastering enough in each domain to excel in integrating diverse data sets for diagnosis of a given case. To a great extent, installation of brain-imaging methods such as EEG, MEG, and fMRI for research purposes remains a cottage industry dependent on individual researchers committing resources to a dense-array EEG system ( $\$ 50 \mathrm{~K}$ and up) or groups of researchers persuading a host institution or funding agency to invest in a research-dedicated MEG or MRI facility (\$2M and up). (PET retains its appeal despite its use of radioisotypes but faces much steeper start-up and support costs, an order of magnitude more.) In the case of MEG, system vendors are prepared to work with entrepreneurial researchers and clinicians to develop a business model for the operation of a facility on the basis of clinical or research billing. The burden often remains, however, on overcommitted individuals to lead such an effort - not an ideal situation for rapid evolution of clinical application capabilities.

It must also be noted that the readiness of neuroimaging for routine clinical use is not a function only of developments in neuroimaging. A separate issue is the marginal practical utility of an expensive diagnostic procedure. The importance and appeal of a diagnostic procedure rests heavily on its implications for treatment. If it were possible, for example, to image exactly the damaged tissue in the brain stem of a patient with Parkinson's disease, at present it would have no implications for treatment or further planning because of limitations in the technology available to treat Parkinson's. The case for clinical neuroimaging will strengthen as progress in other areas helps to justify the assess- ments, to give incremental clinical value to the biological data. In the meantime, there is no insurmountable obstacle to clinical use of these methods right now in principle. Clinicians can approach the effort as an $\mathrm{N}$-of- 1 experiment in a potential clinical case series in line with McFall's (1991) conceptualization of an evolving clinical science, in which experimental procedures can be used alongside established procedures, provided the client or patient provides appropriate consent and the greatest possible scientific rigor is applied.

The research literature on psychological dysfunction using hemodynamic neuroimaging has blossomed in recent years, and space precludes an adequate review. Critical for routine clinical use is establishment of effects with high sensitivity "or specificity, so far nearly absent in the literature. An exception is a report by Bremner (2005) of structural brain imaging successfully discriminating with $100 \%$ accuracy patients with mild Alzheimer's from nonaffected individuals. Functional imaging via MEG, PET, or fMRI is effective in identifying brain regions especially important to preserve (like representational or motor cortex) near areas needing removal when assessment is confined to relatively basic types of function (e.g., Bremner, 2005; Orrison \& Lewine, 1993; J. R. Smith et al., 1995). Figure 2 (from Miller et al., 2006) provides an example of the clinically relevant fine tuning that functional neuroimaging is likely to prove capable of. Heller, Miller, and colleagues have argued that a distinction between types of anxiety can prove valuable not only as a means of distinguishing Diagnostic and Statistical Manual of Mental Disorders diagnoses phenomenologically but as a means of distinguishing brain processes differentially involved in anxiety on the basis of different cognitive processes believed characteristic of the two anxiety types and distinct from those in depression (Heller, Koven, \& Miller, 2003; Heller, Nitschke, Etienne, \& Miller, 1997; Herrington et al., 2006; Levin, Heller, Mohanty, Herrington, \& Miller, in press; Nitschke, Heller, \& Miller, 2000; Nitschke, Heller, Palmieri, \& Miller, 1999). Nonpatients high in self-reported anxious apprehension or anxious arousal and low in anhedonic depression completed a Structured Clinical Interview for DSM-IV Axis I Disorders, Clinician Version (First, Spitzer, Gibbon, \& Williams, 1997) diagnostic session as well as parallel laboratory sessions with EEG recording or fMRI recording while performing color-word and emotional Stroop tasks. The two types of anxiety were associated with different patterns of regional brain activity in line with theory differentiating the two groups on the basis of distinct characteristic processing: With presentation of negative emotional words, the anxious apprehension group preferentially activated Broca's area, reflecting excessive verbal rehearsal, whereas the anxious arousal group preferentially activated a right posterior-temporal area associated with attentional vigilance. Importantly, this differentiation was obtained when comorbid depression was excluded. Although the conceptual specificity of such distinctions may prove important in understanding anxiety at a group level, effect sizes were inadequate to justify use on an individual basis in the clinic. Figure 3 (from Mayberg \& Fossati, in press) provides an impressive example of the richness and complexity of the region-specific models now being developed for psychopathology. Quite a variety of (mostly cortical) regions have been incorporated into this model of brain dysfunction in depression. However, to move the literature beyond demonstrations of group differences with generally insuf- 

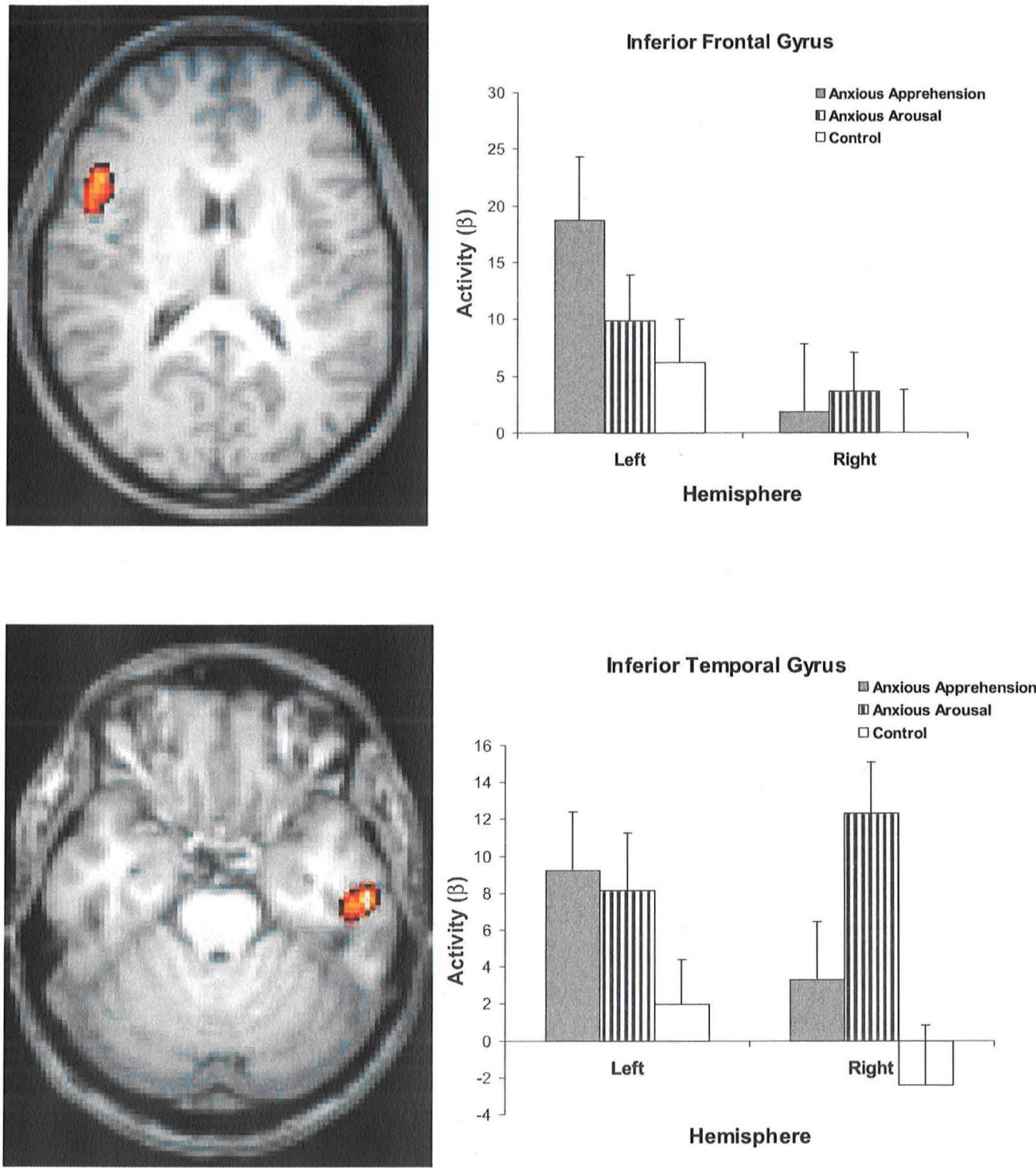

Figure 2. Upper panels: Left inferior frontal gyrus region identified in a nonpatient sample high in anxious apprehension as more active for negative than neutral words in an emotional Stroop experiment (left panel) and mean activations (right panel; mean $\beta$ values, Group $\times$ Hemisphere for the two anxious groups, $p=.010$ ). Lower panels: Right inferior temporal gyrus region identified in a nonpatient sample high in anxious arousal as more active for negative than neutral words (left panel) and mean activations (right panel; mean $\beta$ values, Group $\times$ Hemisphere for the two anxious groups, $p=.002$ ). The left side of the brain is on the left side in the axial slices, and highlighted voxels are those with $z$ scores $>2.58$ meeting cluster-size threshold (based on Engels et al., in press, and Miller et al., 2006).

ficient effect sizes for clinical use, clinicians are going to have to get further involved in the research.

\section{Gaining Entry: How Does a Clinical Psychologist Develop Skills for Brain Imaging?}

It is tempting, but not feasible, to try to lay out areas of expertise that every individual should have to enter the world of neuroimaging. The physics and signal-processing demands of the range of brain-imaging technologies already available are daunting. It is not realistic for most individuals to master psychopathology, clinical neuropsychology, statistical analysis, and the relevant signal processing and biophysics. This type of work must be collaborative. With effort and luck, one identifies talented collaborators to whom one can offer considerable complementary expertise. However, if clinical psychologists leave 


\section{Depression: Limbic-Cortical Dysregulation Model}

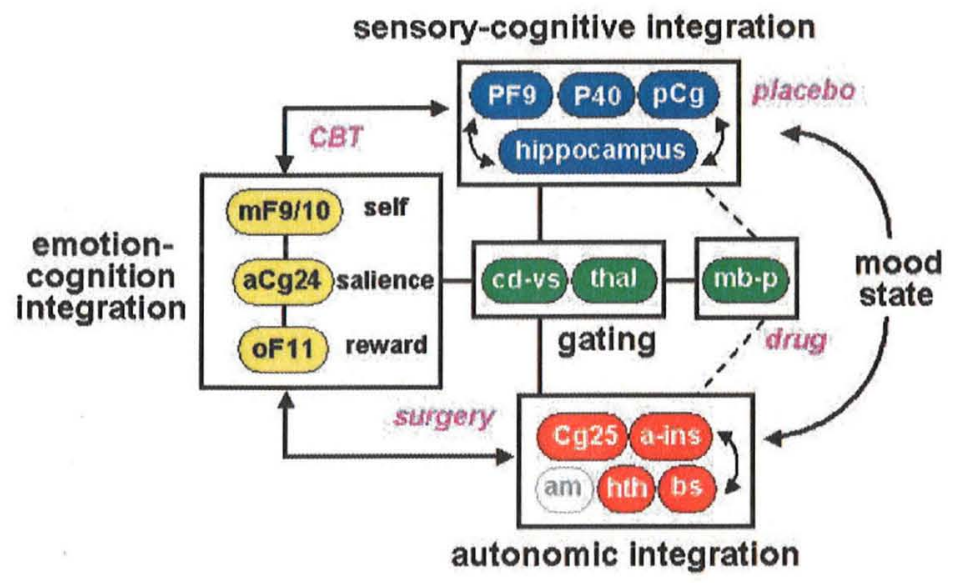

Figure 3. An example of a rich model of psychopathology driven heavily by functional neuroimaging data (Mayberg \& Fossati, in press, adapted from a draft of the published figure with permission from the author; dotted arrows, circles, and labels added). Numbers refer to Brodmann areas. $\mathrm{aCg}=$ rostral anterior cingulated; a-ins $=$ anterior insula; $\mathrm{am}=$ amygdale; $\mathrm{bs}=$ brainstem; CBT $=$ cognitive-behavior therapy; cd-vs $=$ caudate-ventral striatum; $\mathrm{Cg} 25=$ subgenual cingulated; $\mathrm{hth}=$ hypothamus; $\mathrm{mb}-\mathrm{p}=$ midbrain-pons; $\mathrm{mF}=$ medial prefrontal; $\mathrm{oF}=$ orbital frontal; $\mathrm{P} 40=$ inferior parietal; $\mathrm{pCg}=$ posterior cingulated; $\mathrm{PF}=$ dorsolateral, prefrontal; thal $=$ thalamus.

the technical aspects of neuroimaging entirely to their colleagues, they will ensure that translation into the clinic is unnecessarily handicapped.

Because skills in experimental design and data analysis are critical in functional brain imaging research and in clinical applications of it, well-trained clinical psychologists and colleagues in nearby disciplines already have a considerable portion of the skills necessary to use brain-imaging methods-skills relatively lacking in the standard training of many physicists, bioengineers, radiologists, and others already strongly associated historically with brain imaging. Nevertheless, the clinical psychologist considering entry into brain imaging will benefit greatly from whatever relevant physics and signal-analysis training he or she already has had and from additional systematic study of these areas. Those considering substantial clinical or research work using neuroimaging would do well to consider a triage of what expertise they can offer, what skills to invest in developing, and what to rely on colleagues for.

One need not commit to a second $\mathrm{PhD}$. However, one cannot fully leave the physics to the physicists any more than psychologists choose to leave statistics to the statisticians. One has to be able to talk to (and enlighten and persuade) one's collaborators, and one has to be able to read the literature. An onsite physicist or bioengineer is essential at present for first-rate operation of an MEG or MRI facility, particularly for pushing the method beyond established or routine uses. However, such an individual is not a support technician. He or she should be approached as a full collaborator, who can learn as well as teach, with each member of the collaboration actively cross-training. Short courses in fMRI are regularly offered by a number of groups, short courses on EEG and MEG are becoming more common, and technical introductions for the nonspecialist are available (e.g., Cook \& Miller, 1992; Edgar, Stewart, \& Miller, 2005; Herrington, Sutton, \& Miller, in press).

Although psychophysiological imaging methods such as EEG, MEG, fMRI, and optical approaches currently receive the most attention, there are a host of other biological measures, many of them less demanding in hardware cost and signal-processing expertise, more mature in both biological and psychological interpretation, and in some cases already making important contributions to clinical practice, including in behavioral medicine (for selective reviews in psychopathology, see Edgar et al., in press; Keller, Hicks, \& Miller, 2000). The article has focused on noninvasive neuroimaging measures because the rapid rise in their prominence has not been matched in penetration into clinical practice.

The state of the art is almost never ready for routine clinical application. Science is always moving forward, with the potential well ahead of the realization. There is no question that much that is novel and impressive today will be standard clinical practice in the future, some of it in the very near future. Meanwhile, what may be close to ready for application in clinical contexts today is sorely limited by the cultural and technical barriers in the heritage of hemodynamic and electromagnetic psychophysiology as well as typical training in clinical psychology. Indeed, our judgment is that application of neuroimaging technologies in routine clinical practice would be premature. With continued progress, the likelihood is that some disorders will benefit greatly from neuroimaging for diagnosis or tracking of treatment progress, rather than that neu- 
roimaging will become a routine part of assessment in clinical psychology across the board. Individual clinicians may come to believe that some sort of brain scan should be part of a standard battery, just as some have believed that a Rorschach or Structured Clinical Interview for $D S M-I I I-R$ should be almost universal. Among other constraints on such a stance, what one gets from neuorimaging depends heavily on the tasks one gives the subject, very much as in neuropsychological testing. After decades of testing, there is no single, general neuropsychological battery. Some clinicians favor a standard battery, but many choose from a large toolbox on the basis of case-specific hypotheses and symptoms. We anticipate the same approach for neuroimaging.

Psychologists have choices to make about the technical expertise they develop to bring the remarkable clinical potential of these methods to fruition. Rather than wait for the physicists, radiologists, and engineers to figure things out for us, it is essential that clinically oriented psychologists and neuroscientists learn these technologies and help lead the way in clinical application.

Nevertheless, in the face of powerful technologies and seductive illustrations, constant vigilance is needed against the temptation noted at the beginning of this article to mistake brain dysfunction for psychological dysfunction, such as in indefensible but popular claims that mental illness is a brain disorder (or a chemical imbalance or a genetic problem; for extended discussion, see Bennett \& Hacker, 2003; Braitenberg, 1984; Fodor, 1968; Kotchoubey, 2005; Miller, 1996; Miller, Engels, \& Herrington, in press; Miller \& Keller, 2000; Ross \& Pam, 1995). This identification is not feasible on logical grounds, nor is it generative on pragmatic grounds. Naive reductionism unfortunately abounds in the neuroimaging literature and in popular representations of it, where it is often forgotten that psychological rather than biological questions motivate the work. A mental disorder need not be triggered by or due to or explained by brain pathology any more than a software bug must be a consequence of hardware failure. Even when the specific etiology (Meehl, 1973) of a psychological disorder includes brain mechanisms (or gene expression affecting brain mechanisms) in the causal chain, it is untenable to assume that the brain mechanism (or gene expression) is not itself driven by psychological events (Moffitt, 2005; Moffitt et al., 2006). Essential to maximizing the pace and extent of contributions of biological measures to assessment and treatment of varieties of mental illness is remembering that psychological symptoms motivate their assessment and treatment. No advance in biological measurement will alter that.

\section{References}

Bennett, M. R., \& Hacker, P. M. S. (2003). Philosophical foundations of neuroscience. Oxford, England: Blackwell.

Berger, H. (1929). Uber das elektrekephalogramm des menschen [On the human electroencephalogram]. Archives von Psychiatrie und Nervenkranken, 87, 527-570.

Biomedical Information Research Network. (n.d.). Functional imaging research of schizophrenia test bed (function BIRN). Accessed January 13, 2006, from www.nbirn.net/TestBeds/Function/

Bradley, M. M. (Ed.). (2003). Psychophysiology [Entire issue], 40, 487653.

Braitenberg, V. (1984). Vehicles. Cambridge, MA: MIT Press.

Brass, M., Ullsperger, M., Knoesche, T. R., von Cramon, D. Y., \& Phillips, N. A. (2005). Who comes first? The role of the prefrontal and parietal cortex in cognitive control. Journal of Cognitive Neuroscience, 17, 1367-1375.

Bremner, J. D. (2005). Brain imaging handbook. New York: Norton.

Brenner, D., Lipton, J., Kaufman, L., \& Williamson, S. (1978, January 6). Somatically evoked magentic fields of the human brain. Science, 199 , 81-83.

Carey, B. (2005, October 18). Can brain scans see depression? New York Times, pp. D1, D6.

Caton, R. (1875), The electric currents of the brain. British Medical Journal, 2, 278.

Cho, Z. H., \& Ro, Y. M. (1992). Reduction of susceptibility artifact in gradient-echo imaging. Magnetic Resonance in Medicine, 23, 193-200.

Cohen, D. (1968, August 23). Magnetoencephalography: Evidence of magnetic fields produced by alpha rhythm current. Science, 161, 784786.

Cook, E. W., III, \& Miller, G. A. (1992). Digital filtering: Background and tutorial for psychophysiologists. Psychophysiology, 29, 350 -367.

Dale, A. M., \& Buckner, R. L. (1997). Selective averaging of rapidly presented individual trials using fMRI. Human Brain Mapping, 5, 329340.

Dale, A. M., \& Halgren, E. (2001). Spatiotemporal mapping of brain activity by integration of multiple imaging modalities. Current Opinion in Neurobiology, 11, 202-208.

Dale, A. M., \& Sereno, M. I. (1993). Improved localization of cortical activity by combining EEG and MEG with MRI cortical surface reconstruction: A linear approach. Journal of Cognitive Neuroscience, 5, 162-176.

Davis, M., \& Lang, P. J. (2003). Emotion. In M. Gallagher \& R. J. Nelson (Eds.), Handbook of psychology: Vol. 3. Biological psychology (pp. 405-439). Hoboken, NJ; Wiley.

Devor, A., Ulbert, I., Dunn, A. K., Narayanan, S. N., Jones, S. R., Andermann, M. L., et al. (2005). Coupling of the cortical hemodynamic response to cortical and thalamic neuronal activity. Proceedings of the National Academy of Sciences, 102, 3822-3827.

Edgar, J. C., Keller, J., Heller, W., \& Miller, G. A. (in press). Psychophysiology in the study of psychopathology. In J. T. Cacioppo, L. G. Tassinary, \& G. G. Berntson (Eds.), Handbook of psychophysiology (3rd ed.). New York: Cambridge University Press.

Edgar, J. C., Stewart, J. L., \& Miller, G. A. (2005). Digital filtering in EEG/ERP research. In T. C. Handy (Ed.), Event-related potentials: A handbook (pp. 85-113). Cambridge, MA: MIT Press.

Elbert, T. (1998). Neuromagnetism. In W. Andrä \& H. Nowak (Eds.), Magnetism in medicine (pp. 190-262). New York: Wiley.

Engels, A. S., Heller, W., Mohanty, A., Herrington, J. D., Banich, M. T., Webb, A. G., \& Miller, G. A. (in press). Specificity of regional brain activity in anxiety types during emotion processing. Psychophysiology.

Eulitz, C., Elbert, T., Bartenstein, P., Weiller, C., Müller, S. P., \& Pantev, C. (1994). Comparison of magnetic and metabolic brain activity during a verb generation task. NeuroReport, 6, 97-100.

First, M. B., Spitzer, R. L., Gibbon, M., \& Williams, J. B. W. (1997). Structured Clinical Interview for DSM-IV Axis I Disorders (SCID-I), Clinician Version. Washington, DC: American Psychiatric Press.

Fodor, J. A. (1968). Psychological explanation. New York: Random House.

Fuchs, M., Wagner, M., Wischmann, H. A., Ottenberg, K., \& Düssel, O. (1994). Possibilities of functional brain imaging using a combination of MEG and MRT. In C. Pantev, T. Elbert, \& B. Lütkenhöner (Eds.), Oscillatory event related brain dynamics (pp. 435-457). New York: Plenum Press.

Gençer, N. G., Acar, C. E., \& Tanzer, I. O. (2003). Forward problem solution of magnetic source imaging. In Z.-L. Lu \& L. Kaufman (Eds.), Magnetic source imaging of the human brain (pp. 77-100). Mahwah, NJ: Erlbaum.

Glover, G. H. (1999). 3D z-shim method for reduction of susceptibility 
effects in BOLD fMRI. Magnetic Resonance in Medicine, 42, 290299.

Gottesman, I. 1., \& Gould, T. D. (2003). The endophenotype concept in psychiatry: Etymology and strategic intentions. American Journal of Psychiatry, 160, 636-645.

Gratton, G, Fabiani, M., Elbert, T., \& Rockstroh, B. (2003). Seeing right through you: Applications of optical imaging to the study of the human brain. Psychophysiology, 40, 487-491.

Hanlon, F. M., Weisend, M. P., Huang, M., Lee, R. R., Moses, S. N., Paulson, K. M., et al. (2003). A noninvasive method for observing hippocampal function. NeuroReport, 14, 1957-1960.

Hanlon, F. M., Weisend, M. P., Yeo, R. A., Huang, M., Thoma, R. J., Lee, R. R., et al. (2005). A specific test of hippocampal deficit in schizophrenia. Behavioral Neuroscience, 119, 863-875.

Harrison, P. J., \& Weinberger, D. R. (2005). Schizophrenia genes, gene expression, and neuropathology: On the matter of their convergence. Molecular Psychiatry, 10, 40-68.

Harshbarger, T. B., \& Twieg, D. B. (1999). Iterative reconstruction of single-shot spiral MRI with off resonance. IEEE Transactions in Medical lmaging, 18, 196-205.

Heller, W., Koven, N. S., \& Miller, G. A. (2003). Regional brain activity in anxiety and depression, cognition/emotion interaction, and emotion regulation. In K. Hugdahl \& R. J. Davidson (Eds.), The asymmetrical brain (pp. 533-564). Cambridge, MA: MIT Press.

Heller, W., Nitschke, J. B., Etienne, M. A., \& Miller, G. A. (1997). Patterns of regional brain activity differentiate types of anxiety. Journal of Abnormal Psychology, 106, 376-385.

Herrington, J. D., Koven, N. S., Miller, G. A., \& Heller, W. (2006). Mapping the neural correlates of dimensions of personality, emotion, and motivation. In T. Canli (Ed.), Biology of personality and individual differences (pp. 133-156). New York: Guilford Press.

Herrington, J. D., Sutton, B., \& Miller, G. A. (in press). Data-file formats in neuroimaging: Background and tutorial. In J. T. Cacioppo, L. G. Tassinary, \& G. G. Berntson (Eds.), Handbook of psychophysiology (3rd ed.). New York: Cambridge University Press.

Hillyard, S. A., Squires, K. C., Bauer, J. W., \& Lindsay, P. H. (1971, June 25). Evoked potential correlates of auditory signal detection. Science, $172,1357-1360$

Hsu, J. J., \& Glover, G. H. (2005). Mitigation of susceptibility-induced signal loss in neuroimaging using localized shim coils. Magnetic Resonance in Medicine, 53, 243-248.

Huang, M. X., Edgar, J. C., Thoma, R. J., Hanlon, F. M., Moses, S. N., Lee, R. R., et al. (2003). Predicting EEG responses using MEG sources in superior temporal gyrus reveals source anomaly in patients with schizophrenia. Clinical Neurophysiology, 114, 835-850.

Huang, M. X., Shih, J., Lee, R. R., Harrington, D. L., Thoma, R. J., Weisend, M. P., et al. (2004). Commonalities and differences among vectorized beamformers in electromagnetic source imaging. Brain Topography, 16, 139-158.

Huettel, S. A., \& McCarthy, G. (2001a). The effects of aging upon the hemodynamic response measured by functional MRI. Neurolmage, 13, 161-175.

Huettel, S. A., \& McCarthy, G. (200lb). Regional differences in refractory period of the hemodynamic response: An event-related fMRI study. Neurolmage, 14, 967-976.

Huettel, S. A., Song, A. W., \& McCarthy, G. (2004). Functional magnetic resonance imaging. Sunderland, MA: Sinauer Associates.

Hyman, S. E. (2005). Addiction: A disease of learning and memory. American Journal of Psychiatry, 162, 1414-1422.

Jezzard, P., \& Balaban, R. S. (1995). Correction for geometric distortion in echo planar images from $\mathrm{B}_{0}$ field variations. Magnetic Resonance in Medicine, 34, 65-73.

Junghöfer, M., Elbert, T., Tucker, D. M., \& Braun, C. (1999). The polar average reference effect: $A$ bias in estimating the head surface integral in EEG recording. Clinical Neurophysiology, 110, 1149-1155.

Kadah, Y. M., \& Hu, X. (1997). Simulated phase evolution rewinding (SPHERE): A technique for reducing $B_{0}$ inhomogeneity effects in MR images. Magnetic Resonance in Medicine, 38, 615-627.

Keller, J., Hicks, B. D., \& Miller, G. A. (2000). Psychophysiology in the study of psychopathology. In L. G. Tassinary, J. T. Cacioppo, \& G. G Berntson (Eds.), Handbook of psychophysiology (pp. 719-750). New York: Cambridge University Press.

Kendler, K. S. (2005a). "A gene for": The nature of gene action in psychiatric disorders. American Journal of Psychiatry, 162, 1243-1252.

Kendler, K. S. (2005b). Toward a philosophical structure for psychiatry. American Joumal of Psychiatry, I62, 433-440.

Kiebel, S. J., Tallon-Baudry, C., \& Friston, K. J. (2005). Parametric analysis of oscillatory activity as measured with EEG/MEG. Human Brain Mapping, 26, 170-177.

Knake, S., Triantafyllou, C., Wald, L. L., Wiggins, G., Kirk, G. P. Larsson, P. G., et al. (2005). 3T phased array MRI improves the presurgical evaluation in focal epilepsies: A prospective study. Neurology, 11, 1026-1031

Kotchoubey, B. (2005). Neuroscience through the looking glass. Journal of Psychophysiology, 19, 232-237.

Kozak, M. J., \& Miller, G. A. (1982). Hypothetical constructs versus intervening variables: A re-appraisal of the three-systems model of anxiety assessment. Behavioral Assessment, 14, 347-358.

Lang, P. J. (1968). Fear reduction and fear behavior: Problems in treating a construct. In J. M. Shlien (Ed.), Research in psychotherapy (Vol. 3, pp. 90-102). Washington, DC: American Psychological Association.

Lang, P. J. (1978). Anxiety: Toward a psychophysiological definition. In H. S. Akiskal \& W. L. Webb (Eds.), Psychiatric diagnosis: Exploration of biological criteria (pp. 265-389). New York: Spectrum.

Lauterbur, P. D. (1973, March 16). Image formation by induced local interactions: Examples employing nuclear magnetic resonance. Nature 242, 190-191.

Leahy, R. M., Mosher, J. C., Spencer, M. E., Huang, M. X., \& Lewine, J. D. (1998). A study of dipole localization accuracy for $M E G$ and EEG using a human skull phantom (Los Alamos Tech. Rep. No. LA-UR-981442). Los Alamos, NM: Los Alamos National Laboratories.

Levin, R. L., Heller, W., Mohanty, A., Herrington, J. D., \& Miller, G. A. (in press). Cognitive deficits in depression and functional specificity of regional brain activity. Cognitive Therapy and Research

Lewine, J. D., Andrews, R., Chez, M., Devinski, O., Morrell, F., Smith, M., et al. (1999). Magnetoencephalographic patterns of epileptiform activity in children with regressive autism spectrum disorders. Pediatrics, 104, $405-418$.

Lewine, J. D., Davis, J. T., Bigler, E., Hartshorne, M., Thoma, R., Hill, D., et al. (in press). Multimodal brain imaging in mild and moderate head trauma: Integration of MEG, SPECT, and MRI. Journal of Head Trauma Rehabilitation.

Lewine, J. D., Orrison, W. W., Sloan, J. H., Kodituwakku, P. W., \& Davis, J. T. (1999). Neuromagnetic assessment of pathophysiological brain activity induced by minor head trauma. American. Joumal of Neurolog ical Rehabilitation, 20, 857-866.

Li, J., Dietsche, G., Iftime, D., Skipetrov, S. E., Maret, G., Elbert, T,, Rockstroh, B., \& Gisler, T. (2005). Non-invasive detection of functional brain activity with near-infrared diffusing-wave spectroscopy. Journal of Biomedical Optics, 10, 44002-1-12.

Logothetis, N. K., Pauls, J., Augath, M., Trinath, T., \& Oeltermann, A. (2001, July 12). Neurophysiological investigation of the basis of the fMRI signal. Nature, 412, 150-157.

Lütkenhöner, B., Elbert, T., Menninghaus, E., Steinsträter, O., \& Wienbruch, C. (1996). Electro- and magnetoencephalographic source analysis: Current status and future requirements. In H. J. Herrmann, D. E Wolf, \& E. Pöppel (Eds.), Supercomputing in brain research: From 
tomography to neural networks (pp. 175-192). Singapore: World Scientific.

Luu, P., Tucker, D. M., Englander, R., Lockfeld, A., Lutsep, H., \& Oken, B. (2001). Localizing stroke-related EEG changes: Assessing the effects of undersampling. Clinical Neurophysiology, 18, 302-317.

Makeig, S., Delorme, A., Westerfield, M., Jung, T.-P., Townsend, J., Courchesne, E., \& Sejnowski, T. J. (2004). Electroencephalographic brain dynamics following visual targets requiring manual responses. PLOS Biology, 2, 747-762.

Mäkelä, J., Kirveskari, E., Seppä, M., Hämäläinen, M., Forss, N., Avikainen, S., et al. (2001). Three-dimensional integration of brain anatomy and function to facilitate intraoperative navigation around the sensorymotor strip. Human Brain Mapping, 12, 180-192.

Mayberg, H., \& Fossati, P. (in press). Dysfunctional limbic-cortical circuits in major depression: A functional neuroimaging perspective. In $\mathrm{D}$ Barch (Ed.), Cognitive and affective neuroscience of psychopathology. New York: Oxford University Press

McFall, R. M. (1991). Manifesto for a science of clinical psychology. The Clinical Psychologist, 44, 75-88.

McGue, M. (1999). The behavioral genetics of alcoholism. Current Directions in Psychological Science, 8, 109-115.

Meehl, P. E. (1962). Schizotaxia, schizotypy, schizophrenia. American Psychologist, 17, 827-838.

Meehl, P. E. (1973). Specific genetic etiology, psychodynamics, and therapeutic nihilism. In P. E. Meehl (Ed.), Psychodiagnosis: Selected papers (pp. 182-199). Minneapolis: University of Minnesota Press.

Miller, G. A. (1996). Presidential address: How we think about cognition, emotion, and biology in psychopathology, Psychophysiology, 33, 615 628.

Miller, G. A. (2005, September). Neurocognitive mechanisms: Appetive and aversive emotion. Paper presented at National Institute of Drug Abuse symposium, Cognitive Approaches to Addiction: Progress and Pitfalls, Washington, DC.

Miller, G. A., Engels, A. S., \& Herrington, J. D. (in press). The seduction of clinical science: Challenges in psychological and biological convergence. In T. Treat, R. Bootzin, \& R. Levenson (Eds.), Psychological clinical science: Papers in honor of Richard McFall. Mahwah, NJ: Erlbaum.

Miller, G. A., Engels, A. S., Mohanty, A., Herrington, J. D., Levin, R. L., Ho, R. M., Banich, M. T., \& Heller, W. (2006, October). Anxiety type modulates the brain topography of emotion regulation. Paper presented in symposium, Emotion Regulation in Mood and Anxiety Disorders, at the annual meeting of the Society for Research in Psychopathology, San Diego.

Miller, G. A., Herrington, J. D., Mohanty, A., Engels, A. S., Edgar, J. C., Banich, M. T., et al. (2004, October). Frontal-cortex circuitry in emotional self-regulation. Paper presented in symposium, Frontal Lobes and Psychopathology Revisited, at the annual meeting of the Society for Research in Psychopathology, St. Louis.

Miller, G. A., \& Keller, J. (2000). Psychology and neuroscience: Making peace. Current Directions in Psychological Science, 9, 212-215.

Miller, G. A., \& Kozak, M. J. (1993). A philosophy for the study of emotion: Three-systems theory. In N. Birbaumer \& A. Öhman (Eds.), The structure of emotion: Physiological, cognitive and clinical aspects (pp. 31-47). Seattle, WA: Hogrefe \& Huber.

Moffitt, T. E. (2005). The new look of behavioral genetics in developmental psychopathology: Gene-environment interplay in antisocial behaviors. Psychological Bulletin, 131, 533-554.

Moffitt, T. E., Caspi, A., \& Rutter, M. (2006). Measured geneenvironment interactions in psychopathology. Perspectives on Psychological Science, $1,5-27$.

Montague, P. R., Hyman, S. E., \& Cohen, J. D. (2004, October 14). Computational roles for dopamine in behavioural control. Nature, 431, $760-767$.
Moore, K. R., Funke, M. E., Constantino, T., Katzman, G. L., \& Lewine J. D. (2002). Magnetoencephalography directed review of high-spatial resolution surface-coil MR images improves lesion detection in patients with extratemporal epilepsy. Radiology, 225, 880-887.

Mosher, J. C., Spencer, M. E., Leahy, R. M., \& Lewis, P. S. (1993). Error bounds for EEG and MEG dipole source localization. Electroencephalography, 86, 303-321.

Nitschke, J. B., Heller, W., \& Miller, G. A. (2000). Anxiety, stress, and cortical brain function. In J. C. Borod (Ed.), The neuropsychology of emotion (pp. 298-319). New York: Oxford University Press.

Nitschke, J. B., Heller, W., Palmieri, P. A., \& Miller, G. A. (1999). Contrasting patterns of brain activity in anxious apprehension and anxious arousal. Psychophysiology, 36, 628-637.

Nunez, P. L. (2000). Toward a quantitative description of large-scale neocortical dynamic function and EEG. Behavioral and Brain Sciences, 23, 371-437

Ogawa, S., Lee, T. M., Kay, A. R., \& Tank, D. W. (1990). Brain magnetic resonance imaging with contrast dependent on blood oxygenation. Proceedings of the National Academy of Sciences, 87, 9868-9872.

Orrison, W. W., \& Lewine, J. D. (1993). Magnetic source imaging in neurosurgical practice. Perspectives in Neurological Surgery, 4, 141147.

Picton, T. W. (1991). Clinical usefulness of auditory evoked potentials: A critical review. Journal of Speech-Language Pathology and Audiology. 15, 3-18.

Raichle, M. E. (2001, July 12). Cognitive neuroscience: Bold insights. Nature, 412, 128-130.

Reber, P. J., Wong, E. C., Buxton, R. B., \& Frank, L. R. (1998). Correction of off resonance-related distortion in echo-planar imaging using EPIbased field maps. Magnetic Resonance in Medicine, 39, 328-330.

Rockstroh, B., Fehr, T., Kissler, J., Wienbruch, C., \& Elbert, T. (2001). Magnetic source imaging of slow wave activity in psychiatric samples. In J. Nenonen, R. J. Ilmoniemi, \& T. Katila (Eds.), Proceedings of the 12th International Conference on Biomagnetism (Biomag 2000; pp. 395-398). Espoo, Finland: Helsinki University of Technology.

Rockstroh, B., Junghöfer, M., Elbert, T., Guodo, G., \& Miller, G. A. (2006). Evoked magnetic responses indicate deficient automatic processing of affective stimuli in schizophrenia. Psychophysiology, 43, 431439.

Rockstroh, B., Ray, W., Wienbruch, C., \& Elbert, T. (2006). Identification of dysfunctional cortical network architecture and communication: $A b$ normal slow wave activity mapping (ASWAM) in neurological and psychiatric disorders. Manuscript submitted for publication.

Ross, C. A., \& Pam, A. (1995). Pseudoscience in biological psychiatry: Blaming the body. New York: Wiley.

Sabatinelli, D., Bradley, M. M., \& Lang, P. J. (2005). Affective picture processing: Fast functional imaging. Psychophysiology, 42, S6.

Saron, C. D., Horton, D. M., \& Rivera, S. M. (2005, November). Visual, auditory, and somatosensory stimuli in the same perceptual binding domain: A trisensory stimulator for investigating multisensory integration deficits in autism using reaction time and high-density event-related potentials. Paper presented at the Society for Neuroscience meeting, Washington, DC.

Scherg, M., Ille, N., Bornfleth, H., \& Berg, P. (2002). Advanced tools for digital EEG review: Virtual source montages, whole-head mapping, correlation, and phase analysis. Journal of Clinical Neurophysiology, 19, 91-112.

Schneider, E., \& Glover, G. (1991). Rapid in vivo proton shimming. Magnetic Resonance in Medicine, 18, 335-347.

Schomberg, H. (1999). Off-resonance correction of MR images. IEEE Transactions in Medical Imaging, 18, 481-495.

Sekihara, K., Kuroda, M., \& Kohno, H. (1984). Image restoration from 
non-uniform magnetic field influence for direct fourier NMR imaging. Physics in Medicine and Biology, 29, 15-24.

Slotnick, S. D. (2005). Source localization of ERP generators. In T. C. Handy (Ed.), Event-related potentials: A handbook (pp. 149-166). Cambridge, MA: MIT Press.

Smith, J. R., Schwartz, B. J., Gallen, C. C., Orrison, W. W., Lewine, J. D., Murro, A. M., King, D. W., \& Park, Y. D. (1995). Utilization of multichannel magnetoencephalography in the guidance of ablative seizure surgery. Journal of Epilepsy, 8, 119-130.

Smith, S. M., Beckmann, C. F., Ramnani, N., Woolrich, M. W., Bannister, P. R., Jenkinson, M., Matthews, P. M., \& McGonigle, D. J. (2005). Variability in fMRI: A re-examination of inter-session differences. $\mathrm{Hu}$ man Brain Mapping, 24, 248-257.

Srinivasan, R. (2005). High-resolution EEG. In T. C. Handy (Ed.), Eventrelated potentials: A handbook (pp. 167-188). Cambridge, MA: MIT Press.

Stenger, V. A., Boada, F. E., \& Noll, D. C. (2003), Variable-density spiral 3D tailored RF pulses. Magnetic Resonance in Medicine, 50, 1100 1106.

Stöcker, T., Schneider, F., Klein, M., Habel, U., Kellermann, T., Zilles, K., \& Shah, N. J. (2005). Automated quality assurance routines for fMRI data applied to a multicenter study. Human Brain Mapping, 25, 237246.

Sumanaweera, T. S., Glover, G. H., Binford, T. O., \& Adler, J. R. (1993). MR susceptibility misregistration correction. IEEE Transactions in Medical Imaging, 12, 251-259.

Sutton, B. P., Noll, D. C., \& Fessler, J. A. (2003). Fast, iterative, fieldcorrected image reconstruction for MRI. IEEE Transactions in Medical Imaging, 22, 178-188.

Sutton, B. P., Noll, D. C., \& Fessler, J. A. (2004, May). Compensating for within-voxel susceptibility gradients in BOLD fMRI. Proceedings of the I2th Meeting of the International Society of Magnetic Resonance in Medicine, 349.

Swick, D. (2005). ERPs in neuropsychological populations. In T. C. Handy (Ed.), Event-related potentials: A handbook (pp. 299-321). Cambridge, MA: MIT Press.

Tesche, C. D. (1997). Non-invasive detection of ongoing neuronal population activity in normal human hippocampus. Brain Research, 749, $53-60$.

Truong, T. K., Clymer, B. D., Chakeres, D. W., \& Schmalbrock, P. (2002).
Three-dimensional numerical simulations of susceptibility-induced magnetic field inhomogeneities in the human head. Magnetic Resonance in Medicine, 20, 759-770.

Turkheimer, E. (2000). Three laws of behavior genetics and what they mean. Current Directions in Psychological Science, 9, 160-164.

Turner, R., \& Jezzard, P. (1994). Magnetic resonance studies of brain functional activation using echo-planar imaging. In R. W. Thatcher, M. Hallett, T. Zeffiro, E. R. John, \& M. Huerta (Eds.), Functional neuroimaging: Technical foundations (pp. 69-78). San Diego, CA: Academic Press.

Verrotti, A., Pizzella, V., Trotta, D., Madonna, L., Chiarelli, F., \& Romani, G. L. (2003). Magnetoencephalography in pediatric neurology and in epileptic syndromes. Pediatric Neurology, 28, 253-261.

Vieth, J. B., Kober, H., Ganslandt, O., Möller, M., \& Kamada, K. (2003). Clinical applications of brain magnetic source imaging. In Z.-L. Lu \& L Kaufman (Eds.), Magnetic source imaging of the human brain (pp. 343-364). Mahwah, NJ: Erlbaum.

VSM MedTech. (2005, November 4). VSM comments on cut in CMS payment rates for MEG. Retrieved January 6, 2006, from http:// www.ctf.com/news_events/release_details.asp?ID $=331$

Widiger, T. A., \& Clark, L. A. (2000). Toward $D S M-V$ and the classification of psychopathology. Psychological Bulletin, 126, 946-963.

Wilson, J. L., \& Jezzard, P. (2003). Utilization of an intra-oral diamagnetic passive shim in functional MRI of the inferior frontal cortex. Magnetic Resonance in Medicine, 50, 1089-1094.

Wolff, M., Weiskopf, N., Serra, E., Preissl, H., Birbaumer, N., \& Kraegeloh-Mann, I. (2005). Benign partial epilepsy in childhood: Selective cognitive deficits are related to the location of focal spikes determined by combined EEG/MEG. Epilepsia, 46, 1661-1667.

Yang, Q. X., Williams, G. D., Demeure, R. J., Mosher, T. J., \& Smith, M. B. (1998). Removal of local field gradient artifacts in T2*-weighted images at high fields by gradient-echo slice excitation profile imaging. Magnetic Resonance in Medicine, 39, 402-409.

Yudilevich, E., \& Stark, H. (1987). Spiral sampling in magnetic resonance imaging: The effect of inhomogeneities. IEEE Transactions in Medical Imaging, 6, 337-345. 